\title{
ARTICLE Chemogenetic inhibition of lateral habenula projections to the dorsal raphe nucleus reduces passive coping and perseverative reward seeking in rats
}

\author{
Kevin R. Coffey ${ }^{1}$, Ruby E. Marx (iD) ${ }^{1}$, Emily K. Vo ${ }^{1}$, Sunila G. Nair ${ }^{1}$ and John F. Neumaier ${ }^{1}$
}

The lateral habenula $(\mathrm{LHb})$ processes information about aversive experiences that contributes to the symptoms of stress disorders. Previously, we found that chemogenetic inhibition of rat LHb neurons reduced immobility in the forced swim test, but the downstream target of these neurons was not known. Using an intersectional viral vector strategy, we selectively transduced three different output pathways from the LHb by injecting AAV8-DIO-hM 4 Di into the LHb and CAV2-CRE (a retrograde viral vector) into one of the three target areas as follows: dorsal raphe nucleus (DRN), ventral tegmental area (VTA), or rostromedial tegmentum (RMTg). Using the forced swim test, we found that chemogenetic inhibition of DRN-projecting LHb neurons reduced passive coping (immobility), whereas inhibition of the other pathways did not. Chemogenetic activation of DRN-projecting neurons using hM3Dq in another cohort did not further exacerbate immobility. We next examined the impact of inhibiting DRN-projecting LHb neurons on reward sensitivity, perseverative behavior, and anxiety-like behavior using saccharin preference testing, reward-omission testing, and open-field testing, respectively. There was no effect of inhibiting any of these pathways on reward sensitivity, locomotion, or anxiety-like behavior, but inhibiting DRN-projecting LHb neurons reduced perseverative licking during reward-omission testing, whereas activating these neurons increased perseverative licking. These results support the idea that inhibiting LHb projections to the DRN provides animals with resilience during highly stressful or frustrating conditions but not under low-stress circumstances, and that inhibiting these neurons may promote persistence in active coping strategies.

Neuropsychopharmacology (2020) 45:1115-1124; https://doi.org/10.1038/s41386-020-0616-0

\section{INTRODUCTION}

Stress disorders including depression are very common and yet treatment developments have lagged behind our rapidly expanding knowledge about the contribution of various brain regions to the component symptoms of these disorders. One brain region in particular, the lateral habenula $(\mathrm{LHb})$, has received a significant amount of attention in recent years. The $\mathrm{LHb}$ is a highly conserved structure, existing in lamprey, fish, reptiles, and mammals [1]. It receives convergent input from the basal ganglia and the limbic system, and projects to dopaminergic [2], serotonergic, and GABAergic midbrain nuclei [3], making it poised to integrate cognitive and emotional stimuli, and influence behavioral output. The LHb is activated by unexpected negative events and aversive stimuli [4-6], and numerous studies indicate that effective anti-depressants reduce activity in the $\mathrm{LHb}$ and direct inhibition may promote resilience in depressed individuals [7-9]. There is also evidence that $\mathrm{LHb}$ is the primary site of action for the newest Food and Drug Administrationapproved antidepressant, $S(+)$ Ketamine [10]. However, the precise contribution of $\mathrm{LHb}$ to motivated behavior is complex and is an active area of investigation. The LHb has been shown to impact diverse affective processes and behaviors such as attentional regulation [11], positive and negative reward-based decision-making [12-15], flexible and passive coping [16, 17], cognitive flexibility $[18,19]$, and generalized anxiety $[20]$.
Although the medial habenula is organized into circumscribed subregions of neurons with distinct molecular phenotypes, the organization of $\mathrm{LHb}$ has been more difficult to define. Most LHb neurons are glutamatergic [21], but there are numerous neuropeptides and receptors that could contribute to functional specializations of subsets of LHb neurons $[22,23]$. Furthermore, there is no clear subregional organization of gene expression in LHb [24] and neurons expressing transcripts of common interest, such as neuropeptides, monoamine receptors, and potassium channels, tend to be scattered across the LHb. Several reports indicate that $\mathrm{LHb}$ neurons that project to particular target areas such as the dorsal raphe nucleus (DRN), ventral tegmental area (VTA), or rostromedial tegmentum (RMTg) do so without branching to more than one of these targets $[23,25]$. It is not known whether these sets of neurons subserve different functional roles nor whether these pathways diverge in terms of their molecular phenotypes, but given the functional diversity of their targets, we hypothesized that $\mathrm{LHb}$ neurons projecting to these different target areas would have different impacts on stress-associated behaviors.

Given that the neurons projecting to DRN, VTA, or RMTg cannot be selectively targeted with microinjections to subregions and there are no known molecular phenotypes as of yet, we decided to use an intersectional viral vector strategy by injecting Credependent adeno-associated virus (AAV) vectors into the $\mathrm{LHb}$ in combination with a retrograde canine adenovirus-2 (CAV2) Cre

${ }^{1}$ Psychiatry and Behavioral Sciences, University of Washington, 325 9th Avenue, Seattle, WA 98104, USA

Correspondence: John F. Neumaier (neumaier@uw.edu)

Received: 28 October 2019 Revised: 7 January 2020 Accepted: 10 January 2020

Published online: 20 January 2020 
vector into each of these downstream targets. We used chemogenetic inhibition via $\mathrm{hM}_{4} \mathrm{Di}$, a technique that has been well validated in the LHb [26-28], to reveal the distinct contributions of each individual LHb output pathway to stress-associated behaviors. As might be expected, we found that manipulating $\mathrm{LHb}$ neurons impacted behaviors observed only during stressful or frustrating conditions but had no observable impact when the animals were under a low-stress challenge. The LHb-DRN circuit has long been hypothesized to be involved in psychiatric illness [29] and accordingly we found that selectively inhibiting $\mathrm{LHb}$ neurons projecting to DRN increased passive coping associated with immobility during inescapable swim stress and decreased inflexible, perseverative saccharin seeking when the sweet reward was omitted. Conversely, selective activation of the same pathway increased perseverative saccharin seeking. These data suggest $\mathrm{LHb}$ neurons that project to the DRN may be an important target for modulating specific component symptoms of depression.

\section{METHODS}

Experimental design and statistical analyses

Our overarching experimental strategy was to use an intersectional viral vector approach to express DREADDs $\left(\mathrm{hM}_{4} \mathrm{Di}\right.$ or $\left.\mathrm{hM}_{3} \mathrm{Dq}\right)$ in $\mathrm{LHb}$ neurons selectively projecting to the VTA, RMTg, or DRN. This was achieved through injection of Cre-dependent adeno-associated viral vectors (AAV8-hSyn-DIO-hM ${ }_{4}$ Di-mCherry or AAV8-hSynDIO-hM3Dq-mCherry) into the $\mathrm{LHb}$ and a retrogradely transported CAV2-expressing Cre (CAV2-Cre) into one of the three output regions (Fig. 1a). Separate groups of animals were used for each pathway and for each DREADD. In three separate experiments, the effect of inhibiting the LHb's outputs using $\mathrm{hM}_{4} \mathrm{Di}$ was examined during a forced swim test (FST; experiment 1), during saccharin preference testing and reward omission (experiment 2), and during open-field exploration (experiment 3). The effect of activating DRNprojecting $\mathrm{LHb}$ neurons using $\mathrm{hM}_{3} \mathrm{Dq}$ was also tested in all three experimental conditions. All experimental procedures were approved by the University of Washington Institute institutional animal care and use committee, and were conducted in accordance with National Institutes of Health $(\mathrm{NIH})$ guidelines.

We used linear mixed effect modeling (LME; MATLAB) to analyze all behavior. This study required large numbers of animals, meaning that animals receiving DREADDs in different brain regions were run as separate waves. This produced a high level of control within the brain region, but somewhat less control between waves. As such, we first ran LMEs with cohort and treatment as the main effects, and a cohort $\times$ treatment interaction, to determine whether there were differences between behavioral cohorts.

Ime $=$ fitlme $\left(\begin{array}{c}\text { data, 'Time } \sim \text { Region } * \text { Treatment', } \\ \text { 'DummyVarCoding', 'effects' }\end{array}\right) ;$

If such differences existed, separate LMEs were run for each wave, with treatment (Clozapine-N-oxide (CNO) vs. vehicle) as the only fixed effect.

Ime $=$ fitlme $\left(\begin{array}{c}\text { region_data, 'Time } \sim \text { Treatment', } \\ \text { 'DummyVarCoding', 'effects' }\end{array}\right) ;$

Analysis of variances were run on the LMEs to determine the significance and effect sizes were calculated.

Result $=\operatorname{ANOVA}($ Ime $) ; d=$ computeCohen $\_d($ Group A, Group B $)$;

Viral vectors

The Cre-dependent DREADD vectors used in these experiments consisted of AAV (serotype 8) driven by the human synapsin promotor and encoded $\mathrm{hM}_{4} \mathrm{Di}$-mCherry or $\mathrm{hM}_{3} \mathrm{Dq}$-mCherry in double inverted orientation were obtained from Addgene (AAV8hSyn-DIO-hM ${ }_{4}$ Di-mCh and AAV8-hSyn-DIO-hM ${ }_{3}$ Dq-mCh; titers of $\left.\sim 4 \times 10^{12} \mathrm{vg} / \mu \mathrm{l}\right)$. CAV2-CRE was packaged in our laboratory using DKZeo cells [30], purified by $\mathrm{CsCl}_{2}$ step gradient followed by a second $\mathrm{CsCl}_{2}$ purification, and functionally titered using TE26 cells (titer of $\sim 4 \times 10^{10} \mathrm{vg} / \mu \mathrm{l}[31]$ ).

\section{Drugs}

CNO was procured from the NIMH Chemical Synthesis and Drug Supply Program (C-929), and was dissolved in $2 \%$ dimethyl sulfoxide (DMSO) (D8418-50ML, Sigma-Aldrich) and physiological saline solution (18-807-50, Henry Schein Animal Health). CNO solution was prepared at $3 \mathrm{mg} / \mathrm{ml}$ for $1 \mathrm{ml} / \mathrm{kg}$ injections $(0.25-0.5 \mathrm{ml})$. Vehicles were made identically to drug solutions, except CNO was omitted. Cocaine was procured from the NIDA Drug Supply Program and prepared at $10 \mathrm{mg} / \mathrm{ml}$ in $0.9 \%$ sterile saline for $1 \mathrm{ml} / \mathrm{kg}$ injections (0.25-0.5 ml). Meloxicam (6451602845, Henry Schein Animal Health) was prepared at $1 \mathrm{mg} / \mathrm{ml}$ in $0.9 \%$ sterile saline for $1 \mathrm{ml} / \mathrm{kg}$ injections $(0.25-0.5 \mathrm{ml})$.

Experiment 1 (forced swim test)

Male Sprague-Dawley rats ( $n=73$, Charles River) weighing $\sim 250-300 \mathrm{~g}$ were pair housed in a temperature- and humiditycontrolled vivarium on a 12:12 h light-dark cycle and maintained on ad libitum food and water access. For intersectional surgeries, rats were anesthetized with $1-3 \%$ isoflurane. Using a custom robotic stereotaxic instrument [32], 58 animals received $h_{4} \mathrm{Di}$ injected into the LHb. Blunt $28 \mathrm{~g}$ needles were inserted bilaterally at a $10^{\circ}$ angle terminating at $A / P-3.2, M / L \pm 0.7$, and $D / N-5.25$ and $1 \mu$ lo AAV8-hSyn-DIO-hM ${ }_{4}$ Di-mCherry was injected at a rate of $0.2 \mu \mathrm{l} / \mathrm{min}$. Twenty-two animals received bilateral $1 \mu \mathrm{l}$ injections of CAV2-CRE into the DRN at a $15^{\circ}$ angle terminating at $A / P-7.8$, $\mathrm{M} / \mathrm{L} \pm 0.23$, and $\mathrm{D} / \mathrm{N}-6.85$. Eighteen animals received bilateral $1 \mu \mathrm{l}$ injections of CAV2-CRE into the VTA at a $10^{\circ}$ angle terminating at $A / P-5.8, M / L \pm 0.6$, and $D / V-8.6$. Eighteen animals received bilateral $1 \mu$ injections of CAV2-CRE into the RMTg at a $10^{\circ}$ angle terminating at $A / P-7.6, M / L \pm 0.62$, and $D / V-8.5$. Finally, 15 animals received of AAV8-hSyn-DIO-hM ${ }_{3}$ Dq-mCherry in the LHb and CAV2-CRE into the DRN at the same locations and speed. After surgeries, rats were given meloxicam ( $1 \mathrm{mg} / \mathrm{kg}$, subcutaneously (s.c.)) for pain management and monitored for at least 3 days. Accuracy of injection coordinates was confirmed by visualization of mCherry in the $\mathrm{LHb}$; these injection volumes and coordinates were optimized to produce selective transduction of $\mathrm{LHb}$ neurons with minimal expression adjacent regions. Furthermore, the first five surgeries for each output region included $2.5 \%$ by volume CAV2-zsGreen, a direct fluorescence marker of retrograde infection, to ensure the output region coordinates were accurate.

Twenty-one days after the viral infusions, rats were subjected to a FST [33], a procedure commonly used to test the efficacy of antidepressants [34]. Briefly, the animal is placed in an inescapable cylinder containing water $(50 \mathrm{~cm}$ tall, $20 \mathrm{~cm}$ diameter, $30 \mathrm{~cm}$ water depth, $23-25^{\circ} \mathrm{C}$ ) for $15 \mathrm{~min}$ and then removed and dried. The animal is then treated with either vehicle ( $2 \%$ DMSO in saline) or CNO (3 mg/kg intraperitoneally (i.p.); dissolved in $2 \%$ DMSO in saline) three times at 1,5 , and $23.5 \mathrm{~h}$ before the $5 \mathrm{~min}$ post-test swim session, which is performed $24 \mathrm{~h}$ after the first swim. These injection timings were chosen, as they are identical to those used to test traditional anti-depressants in the FST [35]. For strong behavioral control, CNO and vehicle animals were selected from pair-housed males that received viral injections on the same day. These pairs then recovered together and were run through the FST together in side-by-side swim chambers. These paired (CNO and vehicle) swim sessions were recorded from the side at 30 frames per second and rats were tracked using Ethovision XT11 software (Noldus, NL). The tracking data were analyzed using custom MATLAB scripts (Mathworks, Natick MA) to 


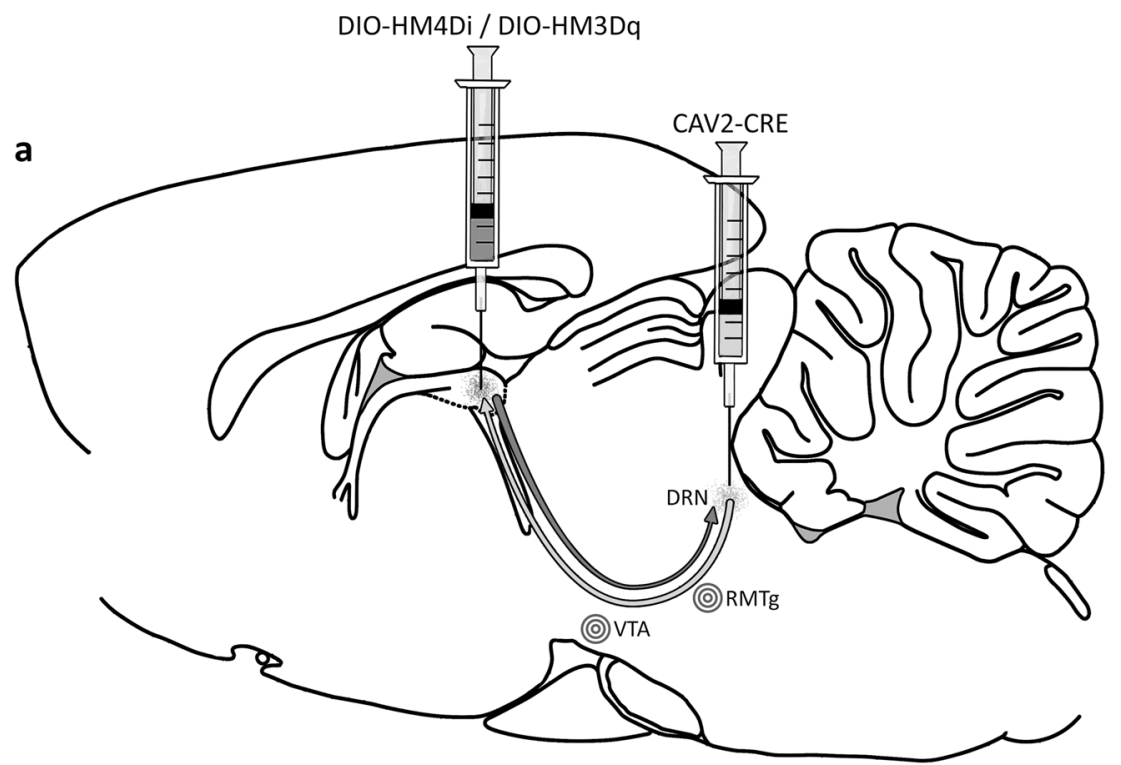

b

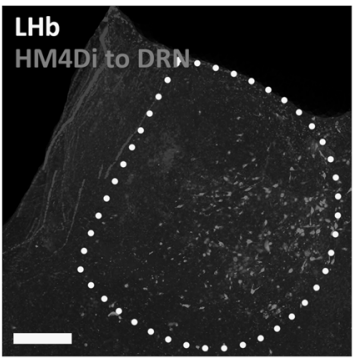

f

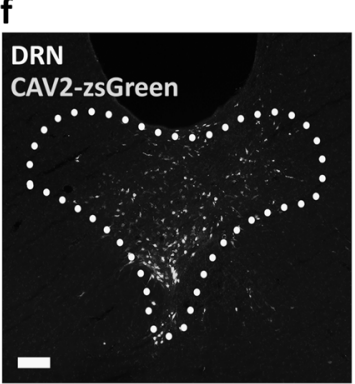

C

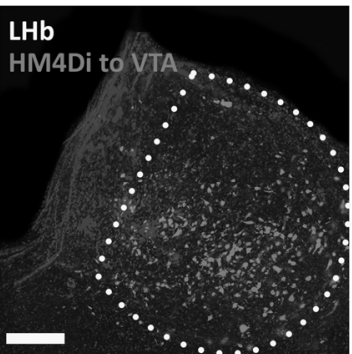

g

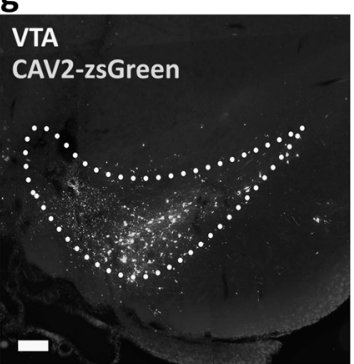

d

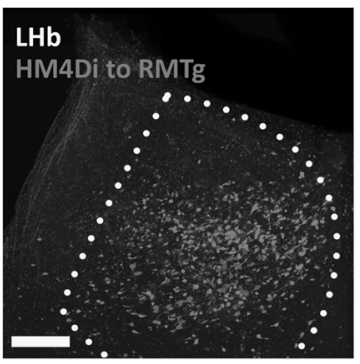

h

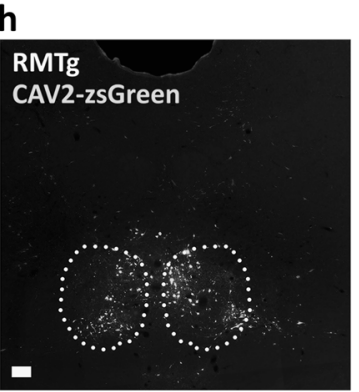

e
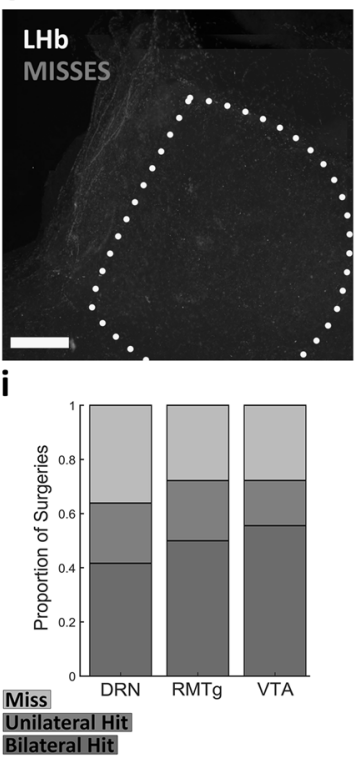

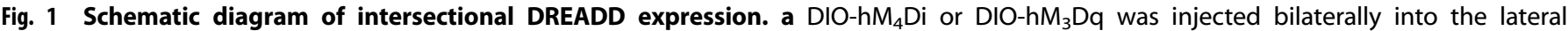
habenula and CAV2-CRE was injected bilaterally into one of the three targeted LHb outputs (VTA, RMTg, or DRN). CAV2-CRE is taken up into terminals and transported retrogradely to $\mathrm{LHb}$ cell bodies where it drives the expression of DREADDs. b-d Maximum intensity projections of DREADD expression in each pathway. One representative slice from each successful surgery in the first experiment (FST) was automatically aligned and overlaid to provide a visual representation of $\mathrm{hM}_{4} \mathrm{Di}$ expression and the anatomical layout of neurons in each pathway. e Maximum intensity projection of LHb slices from "miss" animals. $\mathbf{f}-\mathbf{h}$ A representative injection of CAV2-CRE mixed with 2.5\% CAV2-zsGreen for visualization of terminal targets in the midbrain. i Proportion of surgeries deemed bilateral hit, unilateral hit, or miss. Scale bars $=200 \mu \mathrm{m}$.

determine time spent immobile, defined as movement of $<2 \mathrm{~cm} / \mathrm{s}$. Although the cutoff for movement is technically arbitrary, using an automated approach ensured an unbiased analysis that was applied equally to all subjects.

Experiment 2 (saccharin preference testing)

Male Sprague-Dawley rats ( $n=53$, Charles River) weighing $\sim 250-300 \mathrm{~g}$ were pair housed identically to experiment 1 . Intersectional DREADD expression was performed identically to experiment 1 . Animals $(n=39)$ received $\mathrm{hM}_{4} \mathrm{Di}$ injected into the $\mathrm{LHb}$ as described above. Animals $(n=14)$ received bilateral $1 \mu \mathrm{l}$ injections of CAV2-CRE into the DRN, 13 animals received bilateral $1 \mu \mathrm{l}$ injections of CAV2-CRE into the VTA, and 12 animals received bilateral $1 \mu \mathrm{l}$ injections of CAV2-CRE into the RMTg. A further 14 animals received $\mathrm{hM}_{3} \mathrm{Dq}$ vector in the $\mathrm{LHb}$ and CAV2-CRE into the
DRN at the same locations and speed. After surgeries, rats were given meloxicam $(0.2 \mathrm{mg} / \mathrm{kg}$, s.c.) for pain management and monitored for at least 3 days. At 21 days following viral infusions, rats were tested for saccharin preference using a two-bottle lickometer chamber (Lafayette Instruments, IN) with a water bottle and a bottle of $0.1 \%$ saccharin for $1 \mathrm{~h}$, with a maximum of $35 \mathrm{~mL}$ of saccharin solution and water available. Bottle location was counterbalanced within groups. Animals underwent 4 days of preference training. On the fifth day, animals received either vehicle or $\mathrm{CNO}$ (3 $\mathrm{mg} / \mathrm{kg}$ i.p.) $30 \mathrm{~min}$ prior to saccharin preference testing; this was referred to as the test session. On the sixth day, animals received whichever injection they did not receive on day 5 , either vehicle or CNO (3 mg/kg i.p.). The injection order was randomized and balanced within brain regions. Following the probe sessions, animals underwent 1 more day of normal 
1118

saccharin preference testing on day 7 , followed by a rewardomission session on day 8. During the reward-omission session, animals were randomly assigned to receive either vehicle or CNO ( $3 \mathrm{mg} / \mathrm{kg}$ i.p.) $30 \mathrm{~min}$ prior to the test in which both bottles were filled with water. Licks were counted automatically using an impedance meter connected to each bottle with computerized data collection.

\section{Experiment 3 (open field)}

Male Sprague-Dawley rats from experiment 2 also underwent an open-field exploration procedure 3 days after the end of saccharin preference testing. Thirty minutes prior to the test, animals received either vehicle or CNO (3 mg/kg i.p.). Animals were placed into the center of a $75 \mathrm{~cm}$ diameter circular open field with $65 \mathrm{~cm}$ tall walls. Animals were recorded from the top down against a black floor at $30 \mathrm{fps}$ for $10 \mathrm{~min}$ and were tracked using Ethovision XT11 software (Noldus, NL). Distance traveled, rearing, center time, and center crossings were calculated for each subject.

Validation of $\mathrm{hM}_{4} \mathrm{Di}$ function in the lateral habenula Male, Long-Evans rats $(390-420 \mathrm{~g})(n=8)$ were injected with AAV8-hSyn-hM ${ }_{4}$ Di-mCherry in the $\mathrm{LHb}$ as described above. A separate set of animals $(n=9)$ were injected with AAV8-hSyn-DIO$\mathrm{hM}_{4}$ Di-mCherry in the LHb and CAV2-CRE into the VTA, as described above (Supplementary Fig. S2). Half of the animals in each group were pretreated with either vehicle or CNO $(3 \mathrm{mg} / \mathrm{kg}$, ip). Twenty minutes following pre-treatment all rats were injected with cocaine $(10 \mathrm{mg} / \mathrm{kg}$, i.p.). Ninety minutes following cocaine injections, rats were deeply anesthetized with an i.p. injection of sodium pentobarbital and phenytoin sodium, and were perfused transcardially with $100 \mathrm{ml}$ of $0.1 \mathrm{M}$ phosphate-buffered saline (PBS), followed by $250 \mathrm{ml}$ of cold $4 \%$ paraformaldehyde ( $\mathrm{pH} \mathrm{7.4).}$ Brains were dissected, post fixed in $4 \%$ paraformaldehyde overnight at $4{ }^{\circ} \mathrm{C}$, and transferred to PBS (also at $4{ }^{\circ} \mathrm{C}$ ). Sections $(40 \mu \mathrm{m})$ across the rostro-caudal axis of the $\mathrm{LHb}$ were collected on a Leica VT1000S vibrating blade microtome and stored in $30 \%$ ethylene glycol cryoprotectant until processing.

For cFos immunohistochemistry, free floating sections were rinsed in $1 \times$ PBS $(3 \times, 10 \mathrm{~min})$, permeabilized with $0.5 \%$ Triton PBS, and blocked with $5 \%$ normal goat serum in $0.25 \%$ Triton PBS at room temperature. Sections were incubated with primary antirabbit antibody diluted 1:400 (Abcam; ab87655) in 2.5\% normal goat serum and $0.25 \%$ Triton PBS for $48 \mathrm{~h}$ at $4{ }^{\circ} \mathrm{C}$. Sections were then rinsed with $\mathrm{PBS}(3 \times, 10 \mathrm{~min})$, incubated with 1: 400 dilution of Alexa Fluor Goat anti-rabbit secondary antibody (Invitrogen) for $60 \mathrm{~min}$ at room temperature, washed with PBS $(3 \times 5 \mathrm{~min})$, mounted on gelatin-coated slides, air-dried, and cover-slipped with Pro-Long Gold mounting medium (ThermoFisher Scientific). Cells immunolabeled for cFos were visualized under a Nikon Eclipse E600 microscope and were manually counted in the central $\mathrm{LHb}$ at the bregma level $-3.6 \mathrm{~mm}$.

\section{RESULTS}

Histological verification and localization of DREADD expression One major pitfall of viral-mediated gene transfer is the ability to infect cells outside of the intended target region, potentially confounding the interpretation of results. However, our experience with intersectional expression in $\mathrm{LHb}$ has been more in line with all or nothing expression. As both viruses need to intersect in order to induce DREADD expression in a cell, only neurons that project from $\mathrm{LHb}$ to the target regions can express the transgene. $\mathrm{LHb}$ is surrounded by the medial habenula and thalamus, regions with few, if any, projections to the DRN, RMTg, and VTA. Thus, subjects were included as "hits" if either the left or right LHb contained $\mathrm{HM}_{4} \mathrm{Di}$ or $\mathrm{HM}_{3} \mathrm{Dq}$ expression with at least $90 \%$ of cells confined to the LHb. For experiment 1, of the 72 animals injected with $\mathrm{HM}_{4} \mathrm{Di}$ or $\mathrm{HM} 3 \mathrm{Dq}, 13$ animals were "hits" (4 unilateral and 9 bilateral) for the LHb to DRN pathway, whereas 9 were "misses"; 13 animals were "hits" ( 3 unilateral and 10 bilateral) for the LHb to VTA pathway, whereas 5 were "misses"; 13 animals were "hits" (4 unilateral and 9 bilateral) for the LHb to RMTg pathway, whereas 5 were "misses". Regarding $\mathrm{hM}_{3} \mathrm{Dq}$ experiments, ten animals were "hits" (four unilateral and six bilateral) for the LHb to DRN pathway, whereas four were "misses". Two videos containing data from four animals were corrupted and could not be analyzed. A microscopic view of a single section from each subject included as a hit in this experiment was aligned to the others and was used to produce a maximum intensity projection of the DREADD expression in each pathway (Fig. $1 \mathrm{~b}-\mathrm{h}$ ). DREADD expression using this intersectional technique transduced neurons situated within several hundred micrometers around the injection site. Still, we found that DREADD expression was distributed across the medial-lateral extents of the $\mathrm{LHb}$, regardless of the pathway (Fig. 1b-d). DREADD expression was also apparent in the terminals in VTA, RMTg, and DRN (Supplementary Fig. S1). Targeting of CAV2-CRE was visualized with CAV-ZsGreen and was well-confined to the intended brain target regions (Fig. $1 \mathrm{f}, \mathrm{g}, \mathrm{h}$ ), and collateralization between these pathways is very low, i.e., $0-3 \%$ $[23,36]$. The number of animals that did not show successful intersectional expression of DREADD (i.e., "misses") was relatively high, but these surgeries produced matched control rats for the effect of CNO vs. vehicle on animals without DREADD expression (Fig. 1e), a control that has become necessary in the wake of recent discoveries that $\mathrm{CNO}$ can be back-metabolized to clozapine and have potential off-target effects [37].

Inhibition of DRN-projecting LHb neurons reduces immobility in the forced swim test

Inhibiting DRN-projecting $\mathrm{LHb}$ neurons produces a similar effect to classic anti-depressants in the FST. Animals with $\mathrm{hM}_{4} \mathrm{Di}$ expression in LHb neurons projecting to the DRN and who were administered CNO had significantly decreased immobility compared with vehicle administered animals $(d=1.32 ; \quad F(10,1)=-6.29, \quad p=$ 0.031 ; Fig. $2 b)$. Although immobility in vehicle-treated animals rises steadily throughout the 5 min test swim, immobility in the CNO-treated animals remains low throughout the swim (Fig. 2f). Visualizing activity tracks from these animals exemplifies the increase in swimming and attempts to climb the walls of the swim chamber (Fig. 2j). None of the VTA $(d=0.07$; Fig. 2c), RMTg $(d=$ 0.55 ; Fig. 2d), or Miss ( $d=0.72$; Fig. 2e) groups showed significant differences between $\mathrm{CNO}$ and vehicle animals. Animals in the Miss group came from failed intersectional surgeries from all brain regions and represent a strong control group for the effects of CNO in animals that do not express DREADDs.

Inhibition of DRN-projecting $\mathrm{LHb}$ neurons reduce saccharin licking during a reward-omission test

Animals were trained to lick saccharin in a two-bottle saccharin and water lickometer chamber. All groups of animals learned to increase saccharin licking across four training sessions, with no region by session interaction $(\mathrm{F}(121,3)=7.04, p=0.0002$; Fig. $3 \mathrm{~b}, \mathrm{f}$, $\mathrm{j}, \mathrm{n})$. All animals then received $\mathrm{CNO}$ and vehicle on alternating test sessions to determine whether inhibition of LHb output pathways had any effect on saccharin licking, a hedonic behavior. Inhibition of the LHb outputs to the DRN ( $d=0.04$; Fig. $3 c)$, VTA $(d=0.05$; Fig. $3 g), \operatorname{RMTg}(d=0.18$; Fig. $3 \mathrm{k})$, and Miss ( $d=0.48$; Fig. 3o) did not have a significant effect on saccharin licking. On the final session, saccharin was omitted, two water bottles were presented, and licks on the previously saccharin-paired bottle were measured. Compared with vehicle-treated animals, those with inhibition of DRN-projecting $\mathrm{LHb}$ neurons licked less at the previously saccharin-paired bottle $(d=2.45 ; \quad F(5,1)=-16.00, \quad p=0.007$; Fig. 3d) and licked more at the previously water-paired bottle 

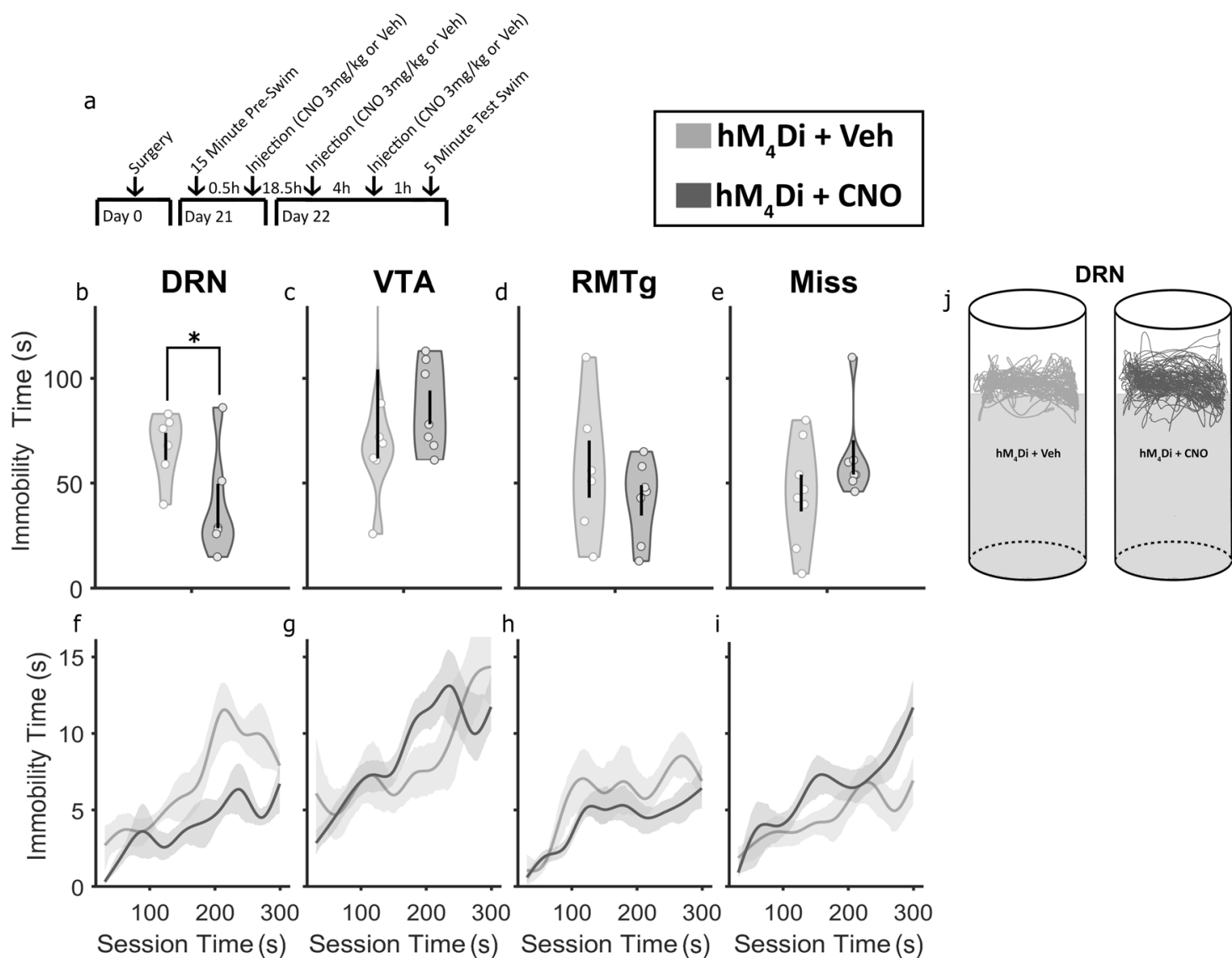

Fig. 2 Inhibition of DRN-projecting LHb neurons reduces immobility in the forced swim test. a Experimental timeline and legend. b Animals with $\mathrm{hM}_{4} \mathrm{Di}$ expression in DRN-projecting LHb neurons, which were administered CNO $(n=6)$, had significantly reduced immobility in the FST compared with vehicle-treated $(n=6)$ animals $(d=1.32 ; \mathrm{F}(10,1)=-6.29, p=0.030)$. c-e CNO had no effect in animals with hM $\mathrm{C}_{4} \mathrm{Di}$ expression in LHb neurons projecting to the VTA (CNO $n=7$, Veh $n=7$; outlier not shown) or RMTg (CNO $n=6$, Veh $n=7)$, or in animals with no DREADD expression (CNO $n=8$, Veh $n=7$ ). $f$ Immobility remained low throughout the FST for animals with hM $\mathrm{H}_{4} \mathrm{Di}$ expression in DRNprojecting LHb neurons, which were administered CNO. g-i Immobility trajectories were not different for CNO- and vehicle-treated animals from all other groups. $\mathbf{j}$ Example swim tracks from two animals with $\mathrm{hM}_{4} \mathrm{Di}$ expression in DRN-projecting LHb neurons. Animals were housed together and run side-by-side. ${ }^{*} p<0.05 ;{ }^{* *} p<0.01$.

$(d=2.02 ; \mathrm{F}(5,1)=10.90, p=0.016 ;$ Fig. 3e). During the rewardomission session, inhibition of the LHb outputs to the VTA ( $d=$ 0.81 ; Fig. 3h), RMTg ( $d=0.52$; Fig. $3 \mathrm{l})$, and Miss ( $d=0.09$; Fig. $3 \mathrm{p})$ did not have a significant effect on previously saccharin-paired bottle. Inhibition of the LHb outputs to the VTA ( $d=0.23$; Fig. 3i), RMTg $(d=0.08$; Fig. $3 \mathrm{~m})$, and Miss $(d=0.72$; Fig. $3 \mathrm{q})$ also did not have a significant effect on water licking. There were no differences in saccharin acquisition between animals prior to being randomly assigned to the $\mathrm{CNO}$ or vehicle groups for reward omission (Supplementary Fig. S3).

Inhibition of LHb neurons projecting to DRN, VTA, and RMTg had no effect on locomotor activity and open-field behavior

After saccharin preference testing, animals were tested in an openfield arena. Open-field testing provided both a control for CNO's effect on locomotion as well as a test of anxiety-like behavior. There were no differences in distance traveled between CNO and vehicle groups for any of the three pathways or the Miss animals (DRN, $d=0.02$; VTA $d=0.84$; RMTg, $d=0.10$; Miss, $d=0.47$; Fig. 4a-d). This is important for interpretation of forced swim behavior, as it shows the reduction in immobility caused by inhibition of the LHb to DRN pathway was not solely due to an increase in general motor activity. There was no evidence of "antianxiety" effects of LHb inhibition in any of the pathways, as evidenced by no significant CNO-induced change in center time (DRN, $d=0.17$; VTA $d=0.36$; RMTg, $d=0.00 ;$ Miss, $d=1.31$;
Fig. 4e-h), center crossings (DRN, $d=0.35$; VTA $d=0.01$; RMTg, $d=0.34$; Miss, $d=0.03$; Fig. $4 \mathrm{i}-1$ ), of rearing (DRN, $d=0.95$; VTA $d=1.26$; RMTg, $d=0.29$; Miss, $d=0.80$; Fig. $4 \mathrm{~m}-\mathrm{p}$ ) for any pathway.

Excitation of DRN-projecting LHb neurons had the opposite effect of inhibition on previously saccharin-paired bottle licking during reward-omission testing

All three experiments were repeated with $h_{3} D q$ expression in the LHb to DRN pathway. Excitation of DRN-projecting LHb neurons had no effect on immobility in the FST $(d=0.50$; Fig. 5a, b) or on saccharin licking during the saccharin preference test $(d=0.13$; Fig. $5 d)$. However, excitation of DRN-projecting LHb neurons increased licking at the previously saccharin-paired bottle during the reward-omission test $(d=2.55 ; \mathrm{F}(8,1)=19.49, p=$ 0.002; Fig. 5e), the opposite effect of chemogenetic inhibition of DRN-projecting LHb neurons. Excitation of DRN-projecting LHb neurons had no effect on licking at the previously water-paired bottle $(d=0.64$; Fig. $5 f)$ or on distance $(d=0.95$; Fig. $5 \mathrm{~g})$, center time $(d=0.71$; Fig. $5 \mathrm{~h})$, center crossings $(d=0.90$; Fig. $5 \mathrm{i})$, or rears $(d=0.59$; Fig. $5 \mathrm{j})$ in the open-field test. The $\mathrm{hM}_{3} \mathrm{Dq}^{*}$ vehicle animals from this experiment had markedly less licking at the previously saccharin-paired bottle then the $\mathrm{hM}_{4} \mathrm{Di}^{*}$ vehicle animals $(257.5 \pm 87.06<658.25 \pm 102.16)$, but the $\mathrm{hM}_{3} \mathrm{Dq}^{*} \mathrm{CNO}$ animals still licked more at the previously saccharin-paired bottle than the $\mathrm{hM}_{4} \mathrm{Di}^{*}$ vehicle animals $(754.67 \pm 74.80>658.25 \pm 102.16)$. 


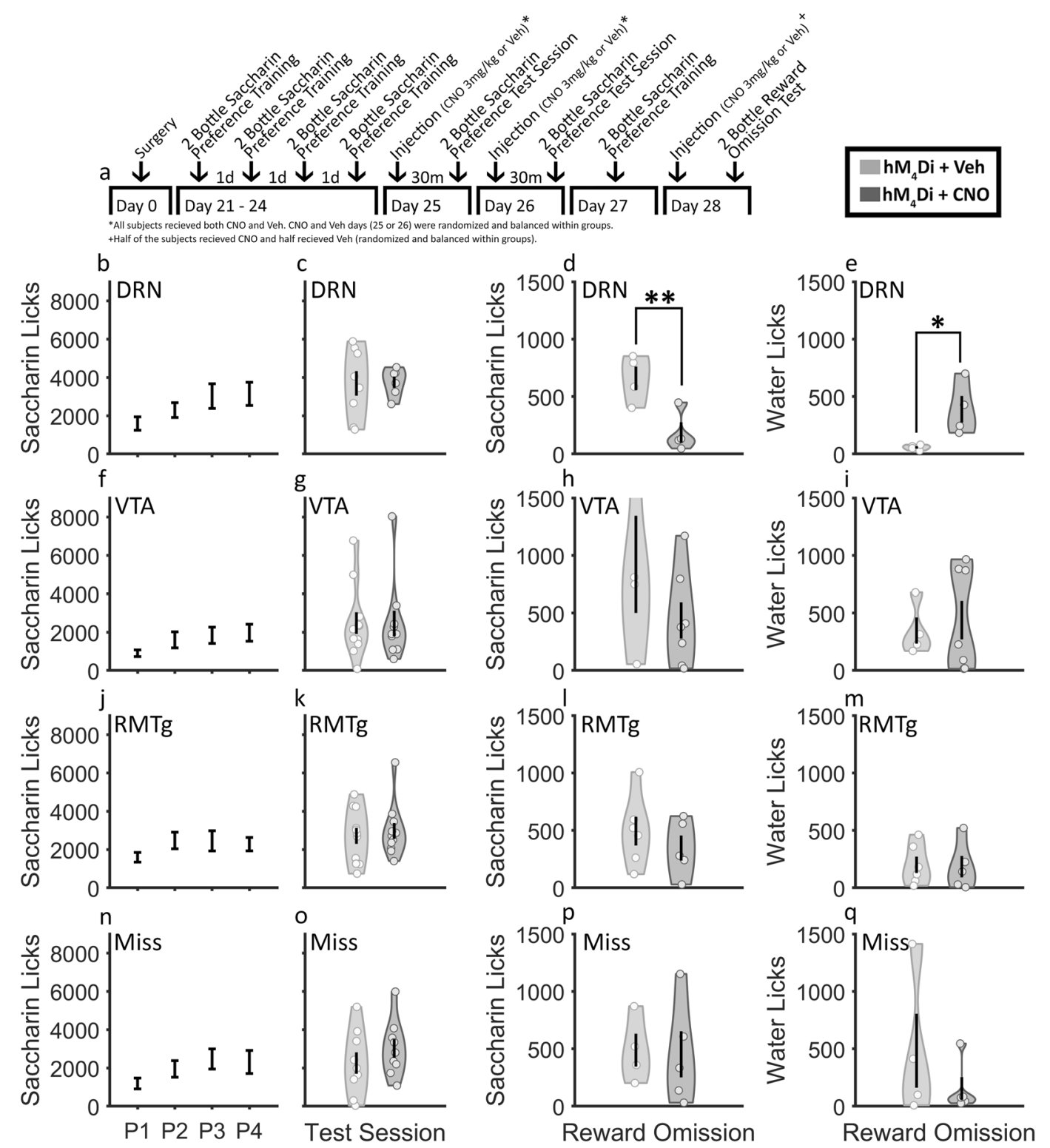

Fig. 3 Inhibition of DRN-projecting LHb neurons decreases perseverative saccharin seeking. a Experimental timeline and legend. $\mathbf{b}$, $\mathbf{f}, \mathbf{j}, \mathbf{n}$ All animals learned to increase saccharin licking across four training sessions in a two-bottle choice lickometer chamber. $\mathbf{d}, \mathbf{h}, \mathbf{I}$ Inhibition of the LHb output pathways (DRN, $n=8$ paired; VTA, $n=11$ paired; RMTg, $n=11$ paired) had no effect on saccharin licking, $\mathbf{p}$ and CNO also had no effect on saccharin licking in animals lacking DREADD receptors $(n=9$, paired). d During a reward-omission test, inhibition of the LHb to DRN pathway reduced licking at a previously saccharin-paired bottle (CNO $n=4$, Veh $n=4 ; d=2.45 ; \mathrm{F}(5,1)=-16.00, p=0.007)$. e During a reward-omission test, inhibition of the LHb to DRN pathway also increased licking at a previously water-paired bottle $(\mathrm{CNO} n=4$, Veh $n=4$; $d=2.02 ; \mathrm{F}(5,1)=-10.90, p=0.016)$. h, i, l, $\mathbf{m}$ Inhibition of LHb outputs to VTA (CNO $n=7$, Veh $n=4$; outlier not shown) and RMTg (CNO $n=6$, Veh $n=5$ ) had no effect on the reward-omission test. p, q CNO also had no effect on reward-omission testing in animals lacking DREADD expression (CNO $n=5$, Veh $n=4$ ). ${ }^{*} p<0.05 ;{ }^{* *} p<0.01$.

Activation of $\mathrm{hM}_{4} \mathrm{Di}$ with $\mathrm{CNO}$ reduces cocaine-induced cFos immunoreactivity in the lateral habenula

To test for $\mathrm{hM}_{4}$ Di-mediated inhibition of $\mathrm{LHb}$ neurons, we stimulated cFos expression with a single injection of cocaine $(10 \mathrm{mg} / \mathrm{kg}$ i.p.) in rats that were pretreated with CNO $(3 \mathrm{mg} / \mathrm{kg}$ i.p.) or vehicle. There was significantly less cocaine-induced $(10 \mathrm{mg} / \mathrm{kg})$ cFos expression (\# of cells) in LHb of rats expressing $\mathrm{hM}_{4} \mathrm{Di}$ and treated with CNO compared with those treated with vehicle $(d=$ 3.02; $t(6)=-24.32 ; p=0.002 ;$ Supplementary Fig. S2c). Similarly, we tested a set of animals with intersectional $\mathrm{hM}_{4} \mathrm{Di}$ expression in $\mathrm{LHb}$ neurons projecting to VTA; again, CNO reduced cFos expression compared with those treated with vehicle $(d=2.66 ; t(5)=-17.049$; $p=0.009$; Supplementary Fig. S2f). Immunohistochemical staining for $\mathrm{hM}_{4} \mathrm{Di}$ failed in these slices, so we were unable to analyze double labeling for DREADD and cFOS, but these results agree with the well-established literature that $\mathrm{hM}_{4} \mathrm{Di}$ stimulation by $\mathrm{CNO}$ can meaningfully reduce activation of LHb neurons [26-28].

\section{DISCUSSION}

The LHb is a phylogenetically conserved structure that acts as a gateway between the limbic system and the monoaminergic midbrain nuclei. This makes the LHb well positioned to integrate complex emotional and motivational signals, and relay this information to the midbrain nuclei responsible for setting the monoaminergic tone of the whole brain. Although the role of the 

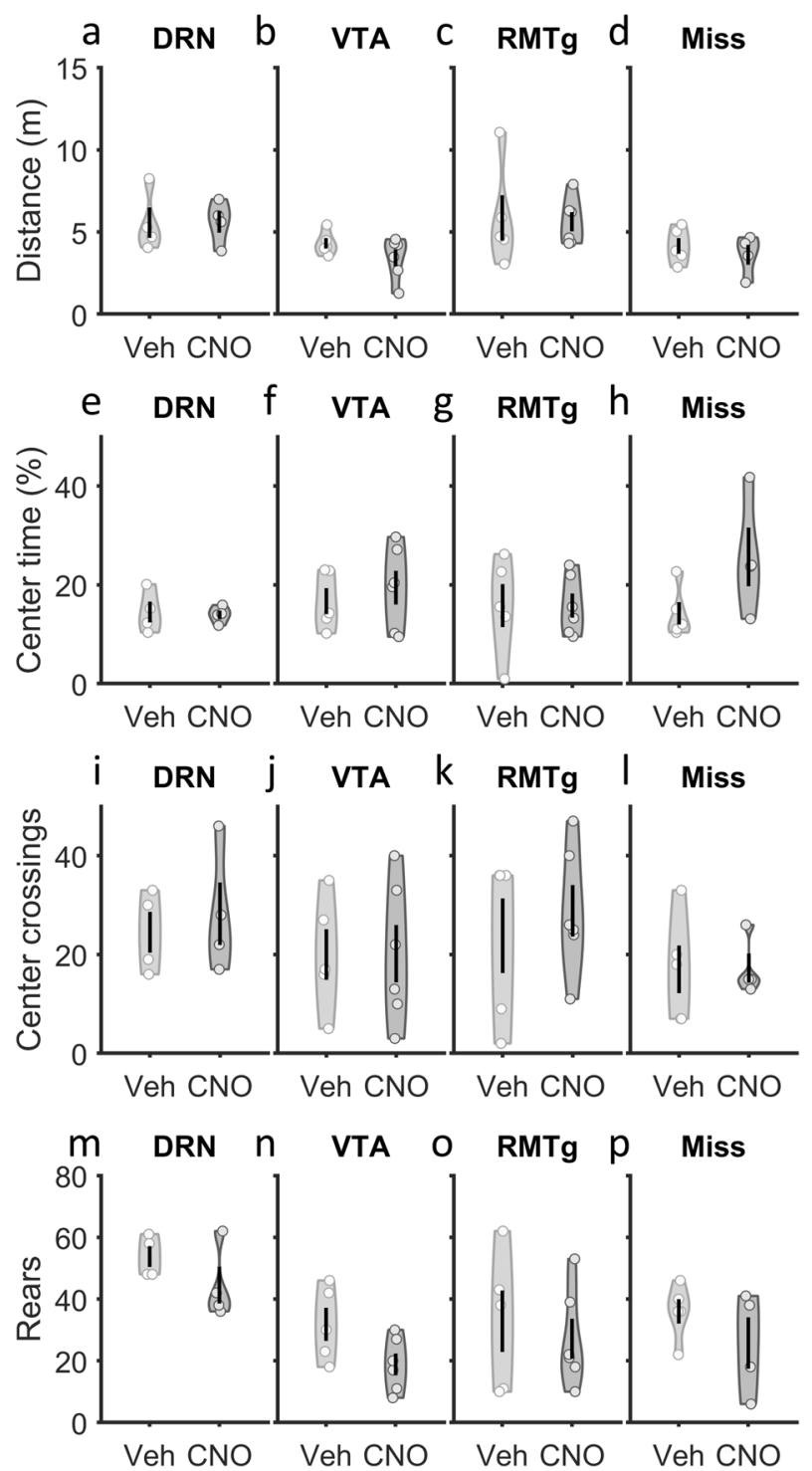

Fig. 4 Inhibition of LHb neurons projecting to DRN, VTA, or RMTg had no effect on locomotor activity or open-field behavior. Inhibition of LHb neurons projecting to DRN (CNO $n=4$, Veh $n=4)$, VTA (CNO $n=6$ Veh $n=5)$, or RMTg (CNO $n=6$, Veh $n=5)$ had no effect on (a, e, i, $\mathbf{m})$ distance traveled, $(\mathbf{b}, \mathbf{f}, \mathbf{j}, \mathbf{n})$ center time, $(\mathbf{c}, \mathbf{g}, \mathbf{k}$, o) center crossings, or (d, $\mathbf{h}, \mathbf{l}, \mathbf{p})$ rearing in the open field. CNO also had no effect on animals without DREADD expression (CNO $n=5$, Veh $n=4$ ).

$\mathrm{LHb}$ in processing aversive stimuli has been well studied as a whole, the relative contributions of its output circuitry to different brain regions are less understood. To probe those circuits, we employed an intersectional viral approach that allowed us to express the inhibitory DREADD $\mathrm{hM}_{4} \mathrm{Di}$ exclusively in neurons that project to DRN, VTA, or RMTg. We then tested a variety of behaviors that induce stress, passive coping, anxiety, reward seeking, and frustration, as each of these can be components of neuropsychiatric disorders such as depression, anxiety, and substance abuse. Our goal was to transiently inhibit or activate the LHb-mediated circuits projecting to these individual midbrain nuclei, thereby altering behaviors associated with those pathologies.

Behavioral testing strategies

We first used the modified Porsolt FST [35]. Each stress model has strengths and limitations, and the FST has been used for more than 30 years and has proved to have excellent predictive validity for antidepressant interventions. For many years, researchers interpreted the rodent's floating response as depressive-like behavior, but this interpretation has fallen out of favor. Swimming and immobility are now seen as the expression of different coping strategies. Although active and passive coping can both be useful survival strategies, drugs that promote active coping tend to improve symptoms of depression in humans, and animals who display a propensity toward active or passive coping have underlying neurobiological differences [38]. We next used the saccharin preference test followed by a reward-omission session. Reduced preference for sweet solutions is a measure of anhedonia that can be tested under low-stress conditions, whereas reward omission assesses coping flexibility in a frustrating condition. Both anhedonia and decreased coping flexibility are associated with clinical depression and anxiety conditions [39], and a decreased resilience following stressful life events $[40,41]$. Finally, we used the open field to test for nonspecific locomotor effects of CNO as well as provide a simple readout of anxiety-like behavior. Overall, we found that inhibition of DRN-projecting LHb neurons may promote active coping and coping flexibility, two important goals in treating depression. This key connection from LHb to DRN has been discussed by previous studies, but had not been directly tested. Inescapable stress induces CFos in DRN-projecting LHb neurons and inhibition of the entire $\mathrm{LHb}$ reduces extracellular 5HT in the basolateral amygdala [42]. Non-intersectional expression of $\mathrm{hM}_{4} \mathrm{Di}$ DREADD receptors and inhibition of LHb neurons also decreased immobility and increased swimming behavior in the FST, a pattern of behavior that also suggested the involvement of the serotonin system [17].

Inhibition of DRN-projecting LHb neurons reduces passive coping in the FST

Lesions or inhibition of the entire $\mathrm{LHb}$ reduces passive coping [7-9] and we previously found that DREADD-mediated inhibition of $\mathrm{LHb}$ neurons had similar antidepressant-like properties [17]. Here we show that $\mathrm{hM}_{4} \mathrm{Di}$-induced inhibition of a subset of $\mathrm{LHb}$ neurons, which project to the DRN was sufficient to elicit this response. Inhibition of DRN-projecting $\mathrm{LHb}$ neurons reduced immobility in the FST, and by attenuating activity in the LHb's glutamatergic projections to the DRN, we can increase resilience to an inescapable stressor by reducing passive coping. CNO concentration in cerebrospinal fluid peaks about 15 min after i.p. injection and is mostly cleared by $60 \mathrm{~min}$, but recent evidence has shown that appreciable levels of back-metabolized clozapine are present in the cerebrospinal fluid at 60 min post injection [43]. We used a standard three-dose protocol to comport with the typical rat FST procedure [33], to allow ready comparisons with previous studies. Our results indicate that the $\mathrm{LHb}$ to DRN projection is critical, but does not determine the exact cognitive mechanism involved; this would require a different battery of behavioral tests to assess whether the animals experience less "helplessness" or an impairment in some aspect of learning. However, we do not believe that this result reflects an impairment in extinction learning. In the reward-omission test, activating $\mathrm{hM}_{4} \mathrm{Di}$ in $\mathrm{LHb}$ projections to DRN decreased licking at the saccharin bottle (faster extinction, if anything) and increased licking at the water bottle, showing a shift in strategy that we interpret as increased coping flexibility.

The LHb's connections to the serotonin system are complex and reciprocal, with $\mathrm{LHb}$ neurons likely synapsing on serotonin neurons, GABAergic neurons, and even glutamatergic neurons [44-46]. There is some conflicting evidence as to what the physiological consequences to serotonin neurons are by inhibiting $\mathrm{LHb}$ inputs. Electrophysiological evidence suggests that $\mathrm{LHb}$ neurons synapse onto DRN GABAergic interneurons, and that inhibiting these neurons would reduce drive on GABA interneurons, thereby disinhibiting serotonin neurons and increasing 

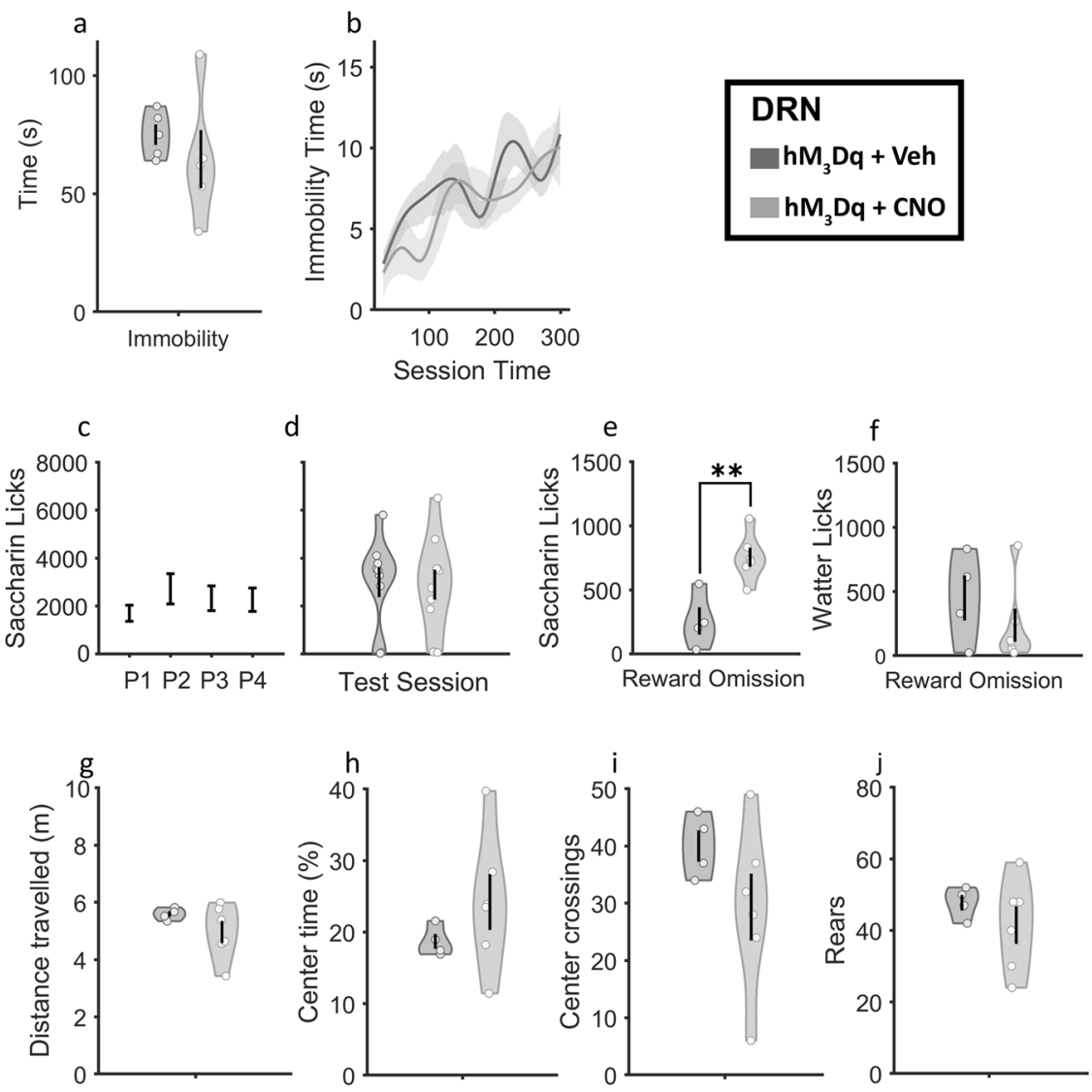

Fig. 5 Excitation of DRN-projecting LHb neurons increases perseverative reward seeking. $\mathbf{a}$, $\mathbf{b}$ Excitation of DRN-projecting LHb neurons (CNO $n=5$, Veh $n=5$ ) had no effect on immobility in the FST. c hM $\mathrm{h}_{3}$ Dq-expressing animals ( $n=10$, paired) learned to increase saccharin licking across four pre-exposures and CNO had no effect on saccharin licking during the test session. e Excitation of DRN-projecting LHb neurons did however increase licking at the previously saccharin-paired bottle during the reward-omission test $(\mathrm{CNO} n=6$, Veh $n=4$; $d=$ 2.55; $F(5,1)=19.49, p=0.002)$, which is the opposite result of inhibiting DRN-projecting LHb neurons. Excitation of DRN-projecting LHb neurons (CNO $n=6$, Veh $n=4$ ) had no effect on $\mathbf{f}$ licking at the previously water-paired bottle, $\mathbf{g}$ distance traveled, $\mathbf{h}$ center time, $\mathbf{i}$ center crossings, or $\mathbf{j}$ rearing in the open field. ${ }^{*} p<0.05 ;{ }^{* *} p<0.01$.

serotonergic tone [47, 48]. GABA injection into the DRN also increases immobility time in the FST and interestingly reduces cFos expression in LHb neurons [49]. This observed decrease in $\mathrm{LHb}$ activity may be due to a negative feedback loop between the DRN and the LHb. Alternatively, there is also evidence that optogenetic silencing of the LHb during inescapable stress blocks increases in extracellular serotonin $(5-\mathrm{HT})$ in the basolateral amygdala [42]. Unfortunately, LHb inhibition in that study was not specific to DRN-projecting neurons and it was difficult to separate the direct effect of inhibiting the DRN-projecting LHb neurons with the effect of inhibiting RMTg-projecting LHb neurons, which are GABAergic and also project to the DRN.

Inhibition of DRN-projecting LHb neurons does not increase general locomotor activity, exploration, or anxiety-like behaviors in the open field, strengthening our interpretation that reduced immobility in the FST represents reduced passive coping rather than a nonspecific enhancement of motor activity. Although it known that the FST induces cFos in the LHb [50], we did not specifically demonstrate that cFos is induced in the LHb to DRN pathway during FST. As we gave CNO after the first swim, we think that decreasing activity in this pathway in the interval between the first and second swim sessions alters plasticity that otherwise develops, but we cannot say with certainty that this pathway was or was not silenced during the second swim session. However, some drugs such as Ketamine and Mibefradil block LHb neuron bursting but do not affect baseline firing, yet are effective in reducing immobility in the FST [10]. Exciting DRN-projecting LHb neurons with $\mathrm{hM}_{3} \mathrm{Dq}$ did not increase immobility in the FST, whereas general $\mathrm{LHb}$ activation can reduce locomotion in an open field but not on the Rotarod [51], so a nonspecific effect on motor activity is unlikely to explain these results. Rather, there may be a ceiling effect for stress-induced excitation of the $\mathrm{LHb}$ that is not exacerbated by $\mathrm{hM}_{3}$ Dq.

Several other recent reports have manipulated $\mathrm{LHb}$ projections to the midbrain in tests designed to probe passive coping or effort exertion. Proulx et al. [52] found that acute optogenetic inhibition of RMTg-projecting LHb neurons transiently decreased immobility during a single FST session, whereas Cerniauskas et al. [36] found that chemogenetic inhibition of LHb neurons projecting to the VTA in mice exposed to 8 weeks of chronic mild stress (CMS) reduced immobility in the tail suspension test. Although on the surface these results do not paint a cohesive story, the fundamental differences between these approaches makes direct comparisons difficult. Proulx et al. [52] utilized a forced swim procedure consisting of alternating 2 min light on/off epochs for RMTg-projecting $\mathrm{LHb}$ neurons during a single $20 \mathrm{~min}$ swim. Optogenetic inhibition causes almost complete silencing of neurons, often followed by rebound excitation during the "off" periods, which may confound data interpretation. This study also did not employ a true intersectional approach, as it expressed inhibitory opsin into LHb non-selectively but administered laser light only into the RMTg. LHb projections to DRN pass through and around the RMTg, meaning they may be inhibited by this manipulation as well. We utilized a truly intersectional technique 
and found that only inhibition of projections to the DRN reduced passive coping. Cerniauskas et al. [36] found that after 8 weeks of CMS, LHb projections to the VTA were sensitized, whereas DRN projections were not. They then show that $\mathrm{hM}_{4} \mathrm{Di}$-based inhibition of $\mathrm{LHb}$ projections to the VTA reduced passive coping in CMS animals, but they did not test inhibition of LHb projections to the DRN. It is possible that CMS changes habenula activity in such a way that makes the projection to the VTA more important for passive coping, whereas our results suggest that during acute stress, the projections to DRN are promoting passive coping. Finally, the current study was only powered sufficiently to find large effect sizes, so modest effects in other pathways may have been missed.

Inhibition and excitation of DRN-projecting $\mathrm{LHb}$ neurons have opposite effects on perseverative reward seeking Inhibiting LHb neurons projecting to the DRN, VTA, and RMTg had no effect on saccharin licking during a saccharin preference test, during which the animal reliably received as much saccharin as it wished, i.e., any frustration associated with the lack of reward is quite low. This is not surprising given that $\mathrm{LHb}$ is generally thought to process primarily aversive stimuli, although unexpected reward delivery can actually inhibit LHb [13]. However, reward omission is known to activate the $\mathrm{LHb}$, suggesting that frustration can be sufficiently aversive to activate $\mathrm{LHb}$, and this may in turn modulate decision-making $[4,15]$. When we inhibited DRN-projecting LHb neurons during reward-omission testing, rats spent less time licking at the previously saccharin-paired bottle. Continued licking at the previously saccharin-paired bottle may represent perseverative reward seeking, whereas promptly reducing effort to obtain an unavailable reward is adaptive. Inhibiting the $\mathrm{LHb}^{\mathrm{S}}$ s projections to the DRN reduced perseverative licking at a bottle previously containing saccharin. This reward-omission condition revealed that frustration activates this pathway and interferes with flexibility, as $\mathrm{hM}_{4}$ Di-mediated inhibition reduced, but $\mathrm{hM}_{3}$ Dq-mediated activation increased, perseverative licking at the bottle previously associated with saccharin. Inability to cope with stressful events flexibly is a correlate of the development of depression in humans $[40,41]$. There was a nonsignificant trend for inhibition of VTAprojecting $\mathrm{LHb}$ neurons to reduce this perseverative licking at the bottle previously associated with saccharin. These results differ from previous reports using pathway nonspecific manipulations of the $\mathrm{LHb}$, but it is particularly difficult to compare pathway-specific manipulations of the $\mathrm{LHb}$ with the global $\mathrm{LHb}$ manipulations, as the regions the $\mathrm{LHb}$ projects to form complex and reciprocal networks both with each other and with the LHb. In either case, the $\mathrm{LHb}$ appears to be important during circumstances requiring flexible, adaptive coping when the likelihood of outcomes is changing $[53,54]$. By alternatively inhibiting or activating DRNprojecting $\mathrm{LHb}$ neurons, our data indicate that this particular pathway plays an important role in flexible coping. Future studies might utilize additional experimental designs that investigate probabilistic learning and responding.

Targeting single circuits with DREADDs may lead to treatments for specific domains of psychiatric illness

Although "sledge hammer" approaches to LHb manipulation (e.g., lesions) cause dramatic effects in aversion and reward processing, DREADD-induced manipulation of individual pathways caused only subtle changes in particular behaviors. In addition to providing a great degree of anatomical precision, chemogenetic approaches allow for transient, bidirectional manipulation of discrete circuits and makes balanced order and within-subjects experimental designs possible. Current treatments for psychiatric illness are plagued by side-effects that are often the root of noncompliance [55]. Our ability to isolate a single circuit with a limited effect on behavior could be crucial to finding treatments with fewer off-target effects. Still, even with our limited subset of behavioral outputs, we found two distinct behaviors that were affected by inhibiting LHb's projections to DRN. Further studies will be needed to tease these behaviors apart and to determine the effect that these manipulations have on the serotonin system. These changes are also highly acute and need to be explored in a chronic setting, as chronic stress in humans is more likely to lead to psychiatric illness than acute stressors.

Data accessibility

The data and code necessary to reproduce the figures and statistics are available at https://github.com/DrCoffey/Manuscripts/ tree/master/LHb\%20Pathways\%20(Neuropsychopharmacology\% 202020) (unpublished).

\section{FUNDING AND DISCLOSURE}

This study was supported by grants R01MH106532, K01DA039999, and T32DA007278. The authors declare no competing interests to disclose.

\section{AUTHOR CONTRIBUTIONS}

$\mathrm{KC}$ performed the experiment, analyzed the data, and wrote the manuscript. RM performed the experiment and analyzed the data. EV performed the experiment. SN performed the experiment and analyzed the data. JN designed the experiment and wrote the manuscript.

\section{ADDITIONAL INFORMATION}

Supplementary Information accompanies this paper at (https://doi.org/10.1038/ s41386-020-0616-0).

\section{REFERENCES}

1. Proulx CD, Hikosaka O, Malinow R. Reward processing by the lateral habenula in normal and depressive behaviors. Nat Neurosci. 2014;17:1146-52.

2. Omelchenko N, Bell R, Sesack SR. Lateral habenula projections to dopamine and GABA neurons in the rat ventral tegmental area. Eur J Neurosci. 2009;30:1239-50.

3. Herkenham M, Nauta WJ. Efferent connections of the habenular nuclei in the rat. J Comp Neurol. 1979;187:19-47.

4. Hikosaka O. The habenula: from stress evasion to value-based decision-making. Nat Rev Neurosci. 2010;11:503-13.

5. Root DH, Mejias-Aponte CA, Qi J, Morales M. Role of glutamatergic projections from ventral tegmental area to lateral habenula in aversive conditioning. J Neurosci. 2014;34:13906-10.

6. Shabel SJ, Proulx CD, Trias A, Murphy RT, Malinow R. Input to the lateral habenula from the basal ganglia is excitatory, aversive, and suppressed by serotonin. Neuron 2012;74:475-81.

7. Gass N, Cleppien D, Zheng L, Schwarz AJ, Meyer-Lindenberg A, Vollmayr B, et al. Functionally altered neurocircuits in a rat model of treatment-resistant depression show prominent role of the habenula. Eur Neuropsychopharmacol. 2014; 24:381-90.

8. Winter C, Vollmayr B, Djodari-Irani A, Klein J, Sartorius A. Pharmacological inhibition of the lateral habenula improves depressive-like behavior in an animal model of treatment resistant depression. Behav Brain Res. 2011;216:463-5.

9. Yang LM, Hu B, Xia YH, Zhang BL, Zhao H. Lateral habenula lesions improve the behavioral response in depressed rats via increasing the serotonin level in dorsal raphe nucleus. Behav Brain Res. 2008;188:84-90.

10. Yang $Y$, Cui $Y$, Sang K, Dong Y, Ni Z, Ma S, et al. Ketamine blocks bursting in the lateral habenula to rapidly relieve depression. Nature 2018;554:317-22.

11. Lecourtier L, Kelly PH. Bilateral lesions of the habenula induce attentional disturbances in rats. Neuropsychopharmacology 2005;30:484-96.

12. Hong S, Jhou TC, Smith M, Saleem KS, Hikosaka O. Negative reward signals from the lateral habenula to dopamine neurons are mediated by rostromedial tegmental nucleus in primates. J Neurosci. 2011;31:11457-71.

13. Matsumoto $M$, Hikosaka $O$. Lateral habenula as a source of negative reward signals in dopamine neurons. Nature 2007;447:1111-5.

14. Ullsperger $M$, von Cramon DY. Error monitoring using external feedback: specific roles of the habenular complex, the reward system, and the cingulate motor area revealed by functional magnetic resonance imaging. J Neurosci. 2003;23:4308-14. 
15. Baker PM, Jhou T, Li B, Matsumoto M, Mizumori SJ, Stephenson-Jones M, et al. The Lateral Habenula Circuitry: Reward Processing and Cognitive Control. J Neurosci. 2016;36:11482-8

16. Lambert KG, Hyer MM, Rzucidlo AA, Bergeron T, Landis T, Bardi M. Contingencybased emotional resilience: effort-based reward training and flexible coping lead to adaptive responses to uncertainty in male rats. Front Behav Neurosci. 2014;8:124.

17. Nair SG, Strand NS, Neumaier JF. DREADDing the lateral habenula: a review of methodological approaches for studying lateral habenula function. Brain Res. 2013;1511:93-101.

18. Lecourtier L, Deschaux O, Arnaud C, Chessel A, Kelly PH, Garcia R. Habenula lesions alter synaptic plasticity within the fimbria-accumbens pathway in the rat. Neuroscience 2006;141:1025-32.

19. Lecourtier L, Neijt HC, Kelly PH. Habenula lesions cause impaired cognitive performance in rats: implications for schizophrenia. Eur J Neurosci. 2004;19:2551-60.

20. Ma Z, Zhong Y, Hines CS, Wu Y, Li Y, Pang M, et al. Identifying generalized anxiety disorder using resting state habenular circuitry. Brain Imaging Behav. 2019; https://doi.org/10.1007/s11682-019-00055-1.

21. Aizawa H, Kobayashi M, Tanaka S, Fukai T, Okamoto H. Molecular characterization of the subnuclei in rat habenula. J Comp Neurol. 2012;520:4051-66.

22. Yang N, Anapindi KDB, Rubakhin SS, Wei P, Yu Q, Li L, et al. Neuropeptidomics of the rat habenular nuclei. J Proteome Res. 2018;17:1463-73.

23. Bernard R, Veh RW. Individual neurons in the rat lateral habenular complex project mostly to the dopaminergic ventral tegmental area or to the serotonergic raphe nuclei. J Comp Neurol. 2012;520:2545-58.

24. Wagner F, French L, Veh RW. Transcriptomic-anatomic analysis of the mouse habenula uncovers a high molecular heterogeneity among neurons in the lateral complex, while gene expression in the medial complex largely obeys subnuclear boundaries. Brain Struct Funct. 2016;221:39-58

25. Brinschwitz K, Dittgen A, Madai Vl, Lommel R, Geisler S, Veh RW. Glutamatergic axons from the lateral habenula mainly terminate on GABAergic neurons of the ventral midbrain. Neuroscience 2010;168:463-76.

26. Kang S, Li J, Zuo W, Chen P, Gregor D, Fu R, et al. Downregulation of M-channels in lateral habenula mediates hyperalgesia during alcohol withdrawal in rats. Sci Rep. 2019;9:2714.

27. Kang S, Li J, Zuo W, Fu R, Gregor D, Krnjevic K, et al. Ethanol withdrawal drives anxiety-related behaviors by reducing M-type potassium channel activity in the lateral habenula. Neuropsychopharmacology 2017;42:1813-24.

28. Tchenio A, Lecca S, Valentinova K, Mameli M. Limiting habenular hyperactivity ameliorates maternal separation-driven depressive-like symptoms. Nat Commun. 2017;8:1135

29. Zhao H, Zhang BL, Yang SJ, Rusak B. The role of lateral habenula-dorsal raphe nucleus circuits in higher brain functions and psychiatric illness. Behav Brain Res. 2015;277:89-98.

30. Soudais C, Boutin S, Kremer EJ. Characterization of cis-acting sequences involved in canine adenovirus packaging. Mol Ther. 2001;3:631-40.

31. Zaiss AK, Son S, Chang LJ. RNA 3 ' readthrough of oncoretrovirus and lentivirus: implications for vector safety and efficacy. J Virol. 2002;76:7209-19.

32. Coffey KR, Barker DJ, Ma S, West MO. Building an open-source robotic stereotaxic instrument. J Vis Exp. 2013;29:e51006.

33. Slattery DA, Cryan JF. Using the rat forced swim test to assess antidepressant-like activity in rodents. Nat Protoc. 2012;7:1009-14.

34. Detke MJ, Rickels M, Lucki I. Active behaviors in the rat forced swimming test differentially produced by serotonergic and noradrenergic antidepressants. Psychopharmacology (Berl). 1995;121:66-72.

35. Porsolt RD, Le Pichon $M$, Jalfre $M$. Depression: a new animal model sensitive to antidepressant treatments. Nature 1977;266:730-2.

36. Cerniauskas I, Winterer J, de Jong JW, Lukacsovich $D$, Yang $H$, Khan $F$, et al. Chronic stress induces activity, synaptic, and transcriptional remodeling of the lateral habenula associated with deficits in motivated behaviors. Neuron 2019;104:899-915.e8.

37. Manvich DF, Webster KA, Foster SL, Farrell MS, Ritchie JC, Porter JH, et al. The DREADD agonist clozapine $\mathrm{N}$-oxide (CNO) is reverse-metabolized to clozapine and produces clozapine-like interoceptive stimulus effects in rats and mice. Sci Rep. 2018;8:3840.

38. Wislowska-Stanek A, Plaznik A, Kolosowska K, Skorzewska A, Turzynska D, LiguzLecznar $M$, et al. Differences in the dopaminergic reward system in rats that passively and actively behave in the Porsolt test. Behav Brain Res. 2019;359:181-9.

39. Cheng $C$. Cognitive and motivational processes underlying coping flexibility: a dual-process model. J Pers Soc Psychol. 2003;84:425-38.

40. Fresco DM, Williams NL, Nugent NR. Flexibility and negative affect: Examining the associations of explanatory flexibility and coping flexibility to each other and to depression and anxiety. Cogn Ther Res. 2006;30:201-10.

41. Gan Y, Liu Y, Zhang Y. Flexible coping responses to severe acute respiratory syndrome-related and daily life stressful events. Asian J Soc Psychol. 2004;7:55-66.

42. Dolzani SD, Baratta MV, Amat J, Agster KL, Saddoris MP, Watkins LR, et al. Activation of a habenulo-raphe circuit is critical for the behavioral and neurochemical consequences of uncontrollable stress in the male rat. eNeuro 2016;3:pii: ENEURO.0229-16.2016

43. Jendryka M, Palchaudhuri M, Ursu $D$, van der Veen B, Liss $B$, Katzel $D$, et al. Pharmacokinetic and pharmacodynamic actions of clozapine-N-oxide, clozapine, and compound 21 in DREADD-based chemogenetics in mice. Sci Rep. 2019;9:4522.

44. Zhou L, Liu MZ, Li Q, Deng J, Mu D, Sun YG. Organization of functional long-range circuits controlling the activity of serotonergic neurons in the dorsal raphe nucleus. Cell Rep. 2017;20:1991-3.

45. Metzger M, Bueno D, Lima LB. The lateral habenula and the serotonergic system. Pharm Biochem Behav. 2017;162:22-8.

46. Sego C, Goncalves L, Lima L, Furigo IC, Donato J Jr., Metzger M. Lateral habenula and the rostromedial tegmental nucleus innervate neurochemically distinct subdivisions of the dorsal raphe nucleus in the rat. J Comp Neurol. 2014;522: 1454-84.

47. Ferraro G, Montalbano ME, Sardo P, La Grutta V. Lateral habenular influence on dorsal raphe neurons. Brain Res Bull. 1996;41:47-52.

48. Varga V, Kocsis B, Sharp T. Electrophysiological evidence for convergence of inputs from the medial prefrontal cortex and lateral habenula on single neurons in the dorsal raphe nucleus. Eur J Neurosci. 2003;17:280-6.

49. Xiao J, Song M, Li F, Liu X, Anwar A, Zhao H. Effects of GABA microinjection into dorsal raphe nucleus on behavior and activity of lateral habenular neurons in mice. Exp Neurol. 2017;298:23-30.

50. Duncan GE, Knapp DJ, Johnson KB, Breese GR. Functional classification of antidepressants based on antagonism of swim stress-induced fos-like immunoreactivity. J Pharm Exp Ther. 1996;277:1076-89.

51. Nair SG, Estabrook MM, Chisholm AD, Silva PR, Neumaier JF. Effect of chemogenetic inhibition of lateral habenula neuronal activity on cocaine- and foodseeking behaviors in the rat. Addict Biol. 2020 (in press).

52. Proulx CD, Aronson S, Milivojevic D, Molina C, Loi A, Monk B, et al. A neural pathway controlling motivation to exert effort. Proc Natl Acad Sci USA. 2018;115: 5792-7.

53. Baker PM, Oh SE, Kidder KS, Mizumori SJ. Ongoing behavioral state information signaled in the lateral habenula guides choice flexibility in freely moving rats. Front Behav Neurosci. 2015;9:295.

54. Baker PM, Raynor SA, Francis NT, Mizumori SJ. Lateral habenula integration of proactive and retroactive information mediates behavioral flexibility. Neuroscience 2017;345:89-98.

55. Blackwell B. Antidepressant drugs: side effects and compliance. J Clin Psychiatry. 1982;43:14-21.

Publisher's note Springer Nature remains neutral with regard to jurisdictional claims in published maps and institutional affiliations.

Springer Nature or its licensor holds exclusive rights to this article under a publishing agreement with the author(s) or other rightsholder(s); author self-archiving of the accepted manuscript version of this article is solely governed by the terms of such publishing agreement and applicable law. 